\title{
Evaluación de la resistencia a la corrosión de recubrimientos obtenidos por pulverización de un blanco de cromo mediante técnicas electroquímicas
}

\section{Evaluation of corrosion resistance on coatings obtained by sputtering of a chromiun cathode by electrochemical techniques}

\author{
Anderson Andrés Sandoval-Amador \\ Físico, Grupo de Investigaciones en Corrosión \\ Universidad industrial de Santander \\ Bucaramanga, Colombia \\ anderson84f@hotmail.com
}

\author{
Darío Yesid Peña-Ballesteros \\ Ph.D. Grupo de Investigaciones en Corrosión \\ Universidad industrial de Santander \\ Bucaramanga, Colombia \\ dypena@uis.edu.co
}

\author{
Ulises Piratona-Morales \\ Ph.D Grupo de Superficies Electroquímica y Corrosión \\ Universidad Pedagógica y Tecnológica de Colombia \\ Tunja, Colombia \\ ulisesp3@hotmail.com
}

\begin{abstract}
Resumen-Se evaluó la resistencia a la corrosión de recubrimientos obtenidos por pulverización de un blanco de cromo sobre sustratos de acero AISI H13, mediante el análisis de espectroscopia de impedancia electroquímica (EIS) y curvas de polarización Tafel en una solución de $\mathrm{NaCl}$ al $3 \%$ en peso. Se encontró que estos recubrimientos presentan una velocidad de corrosión menor a la del sustrato, y que los datos de EIS se ajustan a un circuito eléctrico equivalente que contiene dos elementos de fase constante circuito típico para la interpretación del comportamiento de un electrodo metálico sobre el que ha sido depositado un recubrimiento aislante y poroso.
\end{abstract}

Palabras claves- AISI H13, EIS, pulverización, Tafel, velocidad de corrosión.

Abstract- The corrosion resistance of coatings obtained by sputtering of a chromium target on substrates of steel AISI H13 was evaluated. Through the analysis of electrochemical impedance spectroscopy (EIS) and Tafel polarization curves in a solution of $3 \% \mathrm{NaCl}$ by weight was found that these coatings have a corrosion rate lower than the substrate. Moreover, EIS data fit an equivalent electrical circuit that contains two constant phase elements; this is typical circuit for interpreting the behavior of a metal electrode on which has been deposited an insulating and porous coating.

Keywords- AISI H13, EIS, sputtering, Tafel, corrosion rate.

\section{INTRODUCCIÓN}

Los fenómenos de desgaste y corrosión causan millonarias pérdidas en la industria, generando incrementos en los costos de producción, desfavoreciendo el costo del producto final y los niveles de productividad y competitividad de las compañías [1], [2]. Una alternativa para mitigar estos problemas es la deposición de recubrimientos duros especialmente sobre las herramientas y sobre algunos componentes ingenieriles para protegerlos de la corrosión e incrementar la vida útil de dichos elementos o dispositivos [1], [3], [4]. En este sentido, la deposición física de vapor es una técnica que ha sido ampliamente estudiada en las últimos décadas [5]-[9] y se han producido recubrimientos con base en $\mathrm{CrN}, \mathrm{TiN}, \mathrm{ZrN}$, TiAIN, entre muchos otros [10]-[13]. Estos recubrimientos logran, además, disminuir el coeficiente de fricción, generan un incremento en la estabilidad térmica y la resistencia a la corrosión y al desgaste en diferentes ambientes [14], [15]. De modo que una posible estrategia para mejorar la resistencia a la corrosión del acero H13 es la aplicación de recubrimientos con base en compuestos de nitruros [16]-[18]. 
Para llevar a cabo el estudio de la resistencia a la corrosión en recubrimientos PVD sobre aceros, se han venido implementando algunas técnicas electroquímicas como lo son la espectroscopia de impedancia electroquímica [19] y curvas de polarización Tafel [20] entre otras. El uso de la Espectroscopia de Impedancia Electroquímica (EIS) en la investigación de la corrosión ofrece algunas ventajas respecto a otros métodos electroquímicos, ya que es una técnica prácticamente no destructiva [21] que ofrece información sobre la naturaleza del proceso de corrosión que tiene lugar en la interface metal electrolito; adicionalmente se pueden validar los datos empleando transformaciones de Kramers-Kroning y presentar la interpretación de los mismos mediante modelos de circuitos equivalentes [22]. Por otra parte, las curvas de polarización Tafel del sobrepotencial en función del logaritmo de la corriente, permiten caracterizar el comportamiento de diferentes tipos de metales, identificar el potencial de corrosión y calcular la corriente de corrosión. La técnica Tafel es una técnica rápida pero destructiva por las intensidades de corriente que circulan y desde el punto de vista de la interface, ya que la desordena, lo cual provoca el movimiento de iones que cambian el estado de equilibrio de la interface [20], [23], [24]

\section{MATERIALES Y MÉTODOS}

El acero SISA H13 estudiado fue cortado y maquinado en muestras cilíndricas de $16 \mathrm{~mm}$ de diámetro y $3 \mathrm{~mm}$ de espesor. Todas las probetas fueron desbastadas en una de sus caras y pulidas con papel de carburo de silicio número 120 a 1200 , posteriormente con paños con una solución con partículas suspendidas de alúmina de $3 \mathrm{~mm}$ y $0,05 \mathrm{~mm}$ de diámetro. Finalmente fueron desengrasadas mediante baño ultrasónico en acetona durante 10 minutos. El procedimiento anterior está basado en la norma ASTM G1-03 Standard Practice for Preparing, Cleaning and Evaluating Corrosion Test Specimens.

La composición de los sustratos se identificó mediante un espectrómetro de emisión óptica Bruker Tasman Q4, obteniéndose los resultados que se muestran en la Tabla I. Después, se obtuvieron los recubrimientos mediante la técnica de PVD como se describe en un trabajo previo [25]. Los detalles de los parámetros de trabajo se pueden observar en la Tabla II.

TABLA I

COMPOSICIÓN QUÍMICA DEL ACERO SISA H13 OBTENIDA MEDIANTE ESPECTROMETRÍA DE EMISIÓN ÓPTICA

\begin{tabular}{|c|c|c|c|c|c|}
\hline Elemento & $\mathrm{C}$ & $\mathrm{Si}$ & $\mathrm{Cr}$ & $\mathrm{Mo}$ & $\mathrm{V}$ \\
\hline EEO \% & 0,39 & 1,0 & 5,3 & 1,3 & 0,90 \\
\hline Aceros SISA \% & 0,4 & 1,0 & 5,2 & 1,3 & 0,95 \\
\hline
\end{tabular}

Fuente: autores.

TABLA II

PARÁMETROS DE TRABAJO PARA OBTENCIÓN DE LOS RECUBRIMIENTOS MEDIANTE PVD

\begin{tabular}{|c|c|c|c|c|c|c|c|c|}
\hline & \multicolumn{2}{|c|}{ M1 } & \multicolumn{2}{|c|}{ M2 } & \multicolumn{2}{|c|}{ M3 } & \multicolumn{2}{|c|}{ M4 } \\
\hline Temperatura & \multicolumn{2}{|c|}{$280^{\circ} \mathrm{C}$} & \multicolumn{2}{|c|}{$300^{\circ} \mathrm{C}$} & \multicolumn{2}{|c|}{$296^{\circ} \mathrm{C}$} & \multicolumn{2}{|c|}{$297 \pm 5^{\circ} \mathrm{C}$} \\
\hline \multirow{2}{*}{$\begin{array}{l}\text { Presión } \\
\text { (mbar) }\end{array}$} & Base & Trabajo & Base & Trabajo & Base & Trabajo & Base & Trabajo \\
\hline & 0.16 & 0.34 & 0.17 & 0.38 & 0.18 & 0.38 & 0.19 & 0.37 \\
\hline Voltaje (V) & \multicolumn{2}{|c|}{300} & \multicolumn{2}{|c|}{300} & \multicolumn{2}{|c|}{302} & \multicolumn{2}{|c|}{$335 \pm 5$} \\
\hline Tiempo (h) & \multicolumn{2}{|c|}{4} & \multicolumn{2}{|c|}{4} & \multicolumn{2}{|c|}{3.5} & \multicolumn{2}{|c|}{3.75} \\
\hline Color & \multicolumn{2}{|c|}{ Amarillo } & \multicolumn{2}{|c|}{ Café } & \multicolumn{2}{|c|}{ Gris oscuro } & \multicolumn{2}{|c|}{ Gris oscuro } \\
\hline Gases & \multicolumn{2}{|c|}{ Argon + N2 } & \multicolumn{2}{|c|}{ Argon + N2 } & \multicolumn{2}{|c|}{ Argon + N2 } & \multicolumn{2}{|c|}{ Argon + N2 } \\
\hline
\end{tabular}

Fuente: autores. 
Por último, para la evaluación de la resistencia a la corrosión de los recubrimientos se empleó un potenciostato / galvanostato GAMRY 750 Modelo $\mathrm{PCl}$. El cual se conectaba a una celda electroquímica plana, empleandoAg/AgCl como electrodo de referencia y un electrodo de Platino, como contra electrodo. La solución electrolítica con que se realizaron las pruebas se preparó en agua destilada y desionizada disolviendo $\mathrm{NaCl}$ al $3 \% \mathrm{p} / \mathrm{v}$.

Las pruebas electroquímicas se llevaron a cabo bajo los siguientes parámetros. El rango de frecuencia utilizado para las pruebas de EIS fue de $10 \mathrm{mHz}$ a $1 \mathrm{MHz}$ y se aplicó un potencial de 10 $\mathrm{mV}$ rms. Las curvas de polarización Tafel se realizaron llevando acabo un barrido de $-300 \mathrm{mV}$ hasta $300 \mathrm{mV}$ alrededor del potencial de equilibrio del sistema, que se midió durante 30 minutos, y la velocidad de barrido fue de $1 \mathrm{mV} / \mathrm{s}$.

\section{RESULTADOS}

Las películas estudiadas presentan coloraciones diferentes debido a las variaciones en las variables del proceso de obtención de los recubrimientos, M1 amarillo, M2 café y M3 - M4 gris oscuro (ver Fig. 1). Esta coloración se debe al espesor de las películas obtenidas por el proceso de pulverización catódica y a los compuestos de Cromo o nitruro de cromo que se pudieron formar sobre la superficie del acero. Conviene señalar que a estas probetas se les practicaron ensayos de microdureza para un trabajo anterior [25].

En la Figura 2 se puede observar en las curvas correspondientes a las muestras M1 y M2 que el valor de resistencia del electrolito ha sido inferior a la de las demás probetas. Por otra parte, todas las curvas presentan para bajas frecuencias un aumento de la impedancia con la frecuencia. Este comportamiento se puede modelar incluyendo elementos inductivos; algunos autores [15] atribuyen dichos efectos inductivos a la desorción de impurezas en la superficie de la interface, pero podría obedecer a una reducción de la impedancia por sensibilización de la superficie del metal con el desarrollo del ensayo EIS, ya que se aplican primero las señales de alta frecuencia y se finaliza con las señales de baja frecuencia. Las impurezas adsorbidas podrían ser sitios donde se facilite el intercambio de cargas entre el electrodo metálico y los iones de la solución. Igualmente, se puede observar que las muestras presentaron siempre un valor más alto de impedancia respecto del sustrato.

En la Figura 3 se observa que la curva del sustrato tiene un solo bucle, que implica un único efecto capacitivo, mientras que para las películas, en particular para M2 y M4, se muestra la presencia de dos bucles; esto nos indica que debe emplearse dos elementos de fase constante para el modelo de circuito equivalente y que el recubrimiento aplicado tiene un efecto dieléctrico que aísla y protege la superficie.

Por otra parte, en la Figura 4, diagrama de Nyquist, se puede observar más detalladamente el comportamiento a bajas frecuencias de los componentes imaginarios y reales de la impedancia: se tiene un incremento de las dos partes, este comportamiento se ha reportado en algunas publicaciones [16], pero no se ha publicado un circuito que lo modele; cuando se incluyen elementos inductivos, la parte imaginaria empieza siendo más negativa, y luego aumenta hasta volverse positiva.

Fig. 1. PELÍCULAS OBTENIDAS POR PULVERIZACIÓN DE UN CÁTODO DE CROMO SOBRE ACERO H13. (A) MUESTRA 1- M1, (B) MUESTRA 2 - M2, (C) MUESTRA 3 - M3, (D) MUESTRA 4 - M4

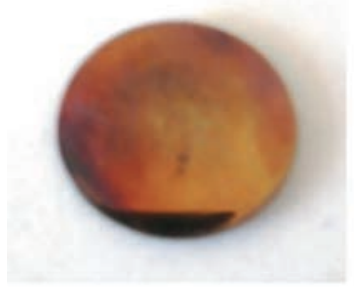

(a

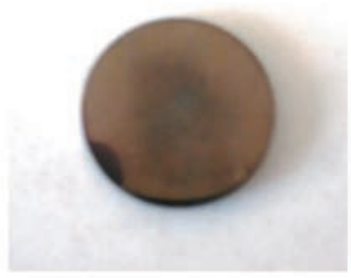

(b

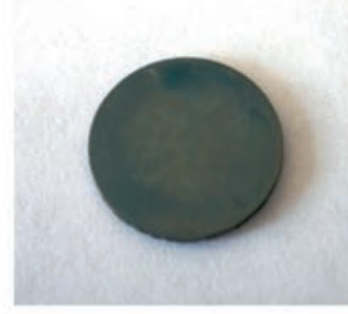

(c

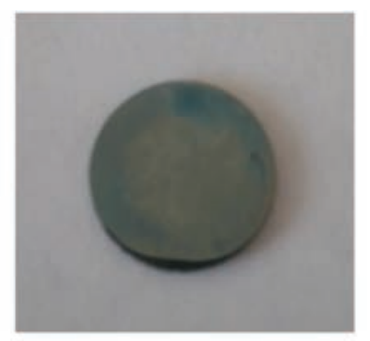

d)

Fuente: autores. 
Fig. 2. DIAGRAMA DE BODE - IMPEDANCIA EN FUNCIÓN DE LA FRECUENCIA

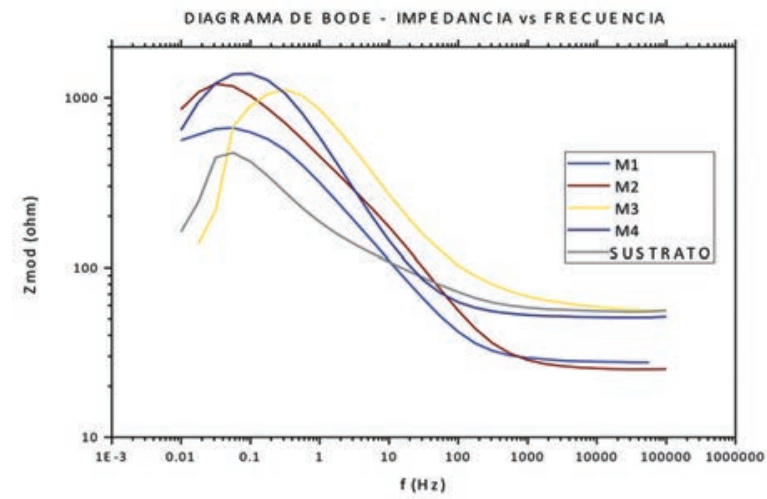

Fuente: autores.

Fig. 4. DIAGRAMA DE NYQUIST - IMPEDANCIA IMAGINARIA EN FUNCIÓN DE LA IMPEDANCIA REAL

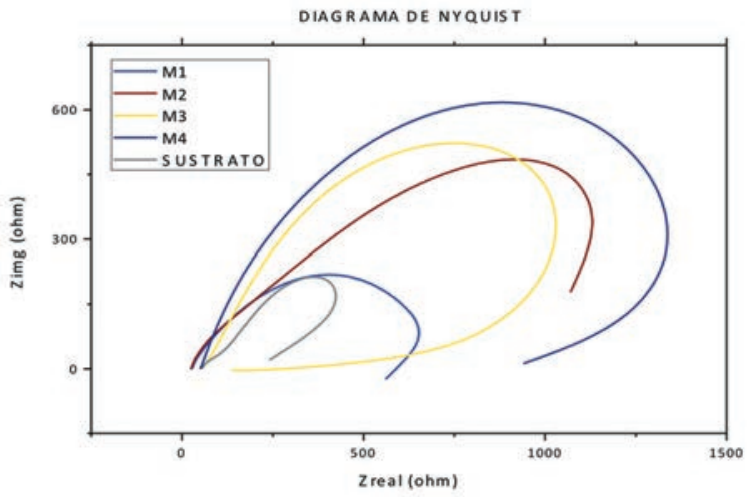

Fuente: autores.

Las curvas de polarización Tafel se presentan en la figura 5; allí podemos observar que para todas las películas los potenciales de corrosión fueron más positivos que el del sustrato, lo que permite afirmar que se mejoró la estabilidad termodinámica de la superficie del metal. Además, al analizar el comportamiento de las corrientes de corrosión, se observa que las probetas recubiertas presentaron valores de $I_{\text {corr }}$ siempre menores que la del sustrato. Las velocidades de corrosión fueron, por tanto, siempre menores en las películas que en el sustrato (ver Tabla III), lo cual indicia que el recubrimiento por PVD de CrN mejoró el comportamiento cinético de las probetas frente a procesos corrosivos. Por otra parte, en las curvas Tafel obtenidas se pudo observar que ninguna de las muestras presentó zonas de pasivación en el rango de sobrepotencial aplicado.
Fig. 3. DIAGRAMA DE BODE - DIFERENCIA DE FASE EN FUNCIÓN DE LA FRECUENCIA

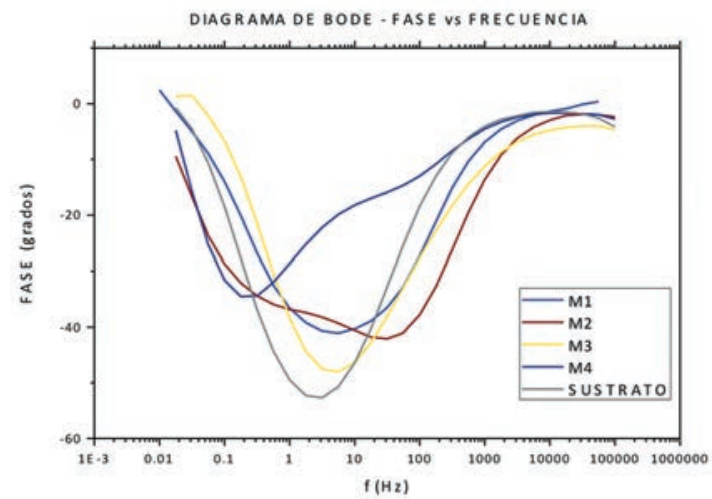

Fuente: autores.

Fig. 5. CURVAS DE POLARIZACIÓN TAFEL

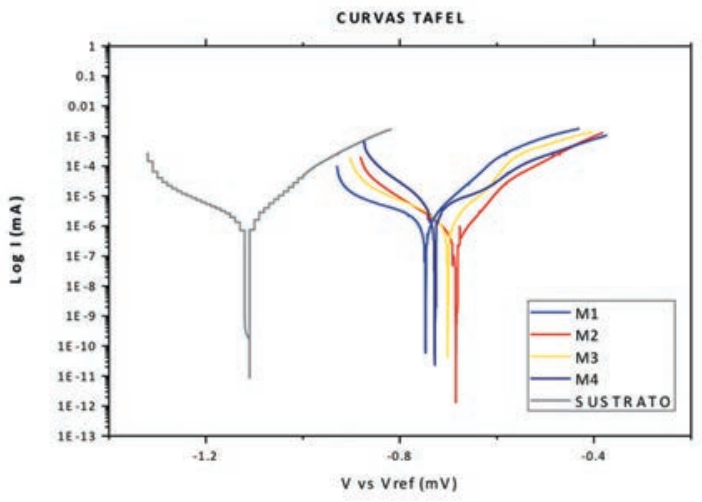

Fuente: autores.

Posteriormente, se procedió a ajustar los comportamientos del sustrato mediante el circuito de Randles mostrado en la Figura 6a, el cual permite modelar sistemas que representan típicamente la interface entre un metal desnudo (sustrato) y el electrolito. Puede representarse mediante la resistencia del electrolito, en serie con el circuito paralelo formado por la resistencia de transferencia de carga con la capacitancia de la doble capa; la capacitancia fue reemplazada por un elemento de fase constante CPE cuya impedancia viene dada por $1 / Y_{0}(j w)^{n}$, donde $Y_{0}$ es una constante pseudocapacitiva; CPE es empleado en los circuitos equivalentes de impedancia electroquímica, por cuanto la respuesta no se ajusta a la de un condensador. Los resultados EIS para las probetas recubiertas se ajustaron al circuito equivalente de la figura $6 \mathrm{~b}$. Este modelo permite describir el com- 
portamiento de una interface electrolito sustrato metálico con recubrimiento aislante que tiene porosidades, donde R1, R2 y R3 son en su orden las resistencias del electrolito, resistencia de poro y resistencia a la transferencia de carga. Posee 2 elementos de fase constante, uno representa la pseudocapacitancia de la doble capa CEP1 $\left(\mathrm{Y}_{\mathrm{dl}}\right)$ y otro representa la pseudocapacitancia del recubrimiento CPE2 $\left(\mathrm{Y}_{\mathrm{c}}\right)$.

Fig. 6. MODELOS DE CIRCUITO EQUIVALENTE

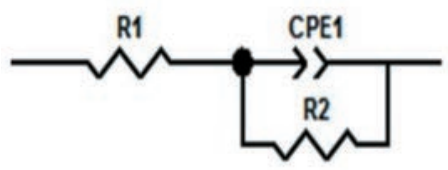

a)

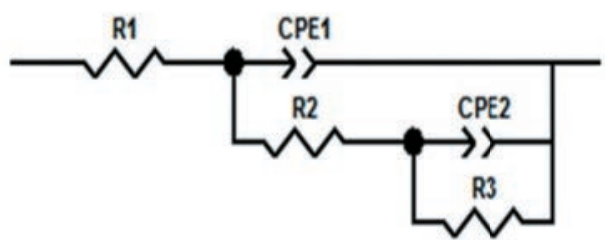

b)

Fuente: autores.

De los parámetros indicados en las Tablas IV y $\mathrm{V}$ se puede observar que las resistencias del electrolito tienen el mismo orden de magnitud y son muy similares; las resistencias de poro varían entre unos 35 y 218 ohmios. Se obtuvo para la probeta M4 la mayor resistencia de poro y resistencia a la transferencia de carga. Los máximos valores de las dos pseudocapacitancias se obtuvieron para la probeta M4, para la cual y como ya se mencionó antes, se obtuvo también la velocidad de corrosión más baja; estos resultados podrían estar mostrando que la capa superficial modificada o depositada con el tratamiento es muy delgada, ofrece una protección efectiva al metal, pero que también hay una penetración significativa del electrolito al recubrimiento y hace contacto con el metal.

\section{CONCLUSIONES}

Los resultados obtenidos muestran que los recubrimientos de nitruro de cromo exhiben una mayor resistencia a la corrosión comparado con el del sustrato, y pese a que las curvas de polarización Tafel obtenidas no mostraron zonas de pasivación, estas permitieron apreciar que el tratamiento superficial aumenta la estabilidad termodinámica del material, disminuyendo las corrientes y velocidades de corrosión. Además, los diagramas de impedancia mostraron para el sustrato el comportamiento típico de un metal desnudo en contacto con un electrolito, y para los sustratos con recubrimiento, un comportamiento similar al de un metal con revestimiento aislante en contacto con un electrolito. Esto permite inferir que se pudieron obtener recubrimientos de cromo o de nitruro de cromo obtenidos por PVD al bombardear el blanco de cromo y que estos mejoran las propiedades electroquímicas y la vida útil del acero SISA $\mathrm{H} 13$.

TABLA III

PARÁMETROS OBTENIDOS DE LA CURVAS DE POLARIZACIÓN TAFEL

\begin{tabular}{|c|c|c|c|c|c|}
\hline Parámetro & $\begin{array}{c}\text { Beta } \mathrm{A} \\
(\mathrm{V} / \mathrm{decada})\end{array}$ & $\begin{array}{c}\text { Beta C } \\
(\mathrm{V} / \text { decada })\end{array}$ & Ecorr $(\mathrm{mV})$ & Icorr $(\mu \mathrm{A})$ & $\begin{array}{c}\text { Velocidad } \\
\text { de Corrosión } \\
(\mathrm{mpy})\end{array}$ \\
\hline Sustrato & $6.03 \mathrm{E}-02$ & $1.03 \mathrm{E}-01$ & -1110 & 1.530 & $7.98 \mathrm{E}-01$ \\
\hline $\mathrm{M} 1$ & $6.91 \mathrm{E}-02$ & $1.70 \mathrm{E}-01$ & -747 & 0.692 & $3.53 \mathrm{E}-01$ \\
\hline $\mathrm{M} 2$ & $4.57 \mathrm{E}-02$ & $7.28 \mathrm{E}-02$ & -683 & 0.21 & $1.07 \mathrm{E}-01$ \\
\hline $\mathrm{M} 3$ & $6.47 \mathrm{E}-02$ & $1.22 \mathrm{E}-01$ & -701 & 1.130 & $5.75 \mathrm{E}-01$ \\
\hline $\mathrm{M} 4$ & $6.72 \mathrm{E}-02$ & $4.61 \mathrm{E}-02$ & -727 & 0.353 & $1.80 \mathrm{E}-01$ \\
\hline
\end{tabular}


TABLA IV

PARÁMETROS DE AJUSTE MEDIANTE EL CIRCUITO DE LA FIGURA 6a PARA EL SUSTRATO

\begin{tabular}{|c|c|}
\hline Parámetro & Valor \\
\hline $\mathrm{R} 2(\Omega)$ & $1.663 e+3$ \\
\hline $\mathrm{R} 1(\Omega)$ & 51.19 \\
\hline $\mathrm{YO}(\mathrm{S} * \mathrm{~s} \alpha)$ & $370.3 e-6$ \\
\hline Alpha $(\alpha)$ & 0.765 \\
\hline
\end{tabular}

Fuente: autores.

TABLA V

PARÁMETROS DE AJUSTE PARA LAS PELÍCULAS MEDIANTE EL CIRCUITO DE LA FIGURA 6b

\begin{tabular}{|c|c|c|c|c|c|c|c|}
\hline Parámetro & Rporo $(\Omega)$ & $\operatorname{Rtc}(\Omega)$ & $\operatorname{Re}(\Omega)$ & Ydl $\left(\mathrm{S}^{*} \mathrm{~S}^{\wedge} \mathrm{Ndc}\right)$ & $\mathrm{Ndl}$ & $\mathrm{Yc}\left(\mathrm{S}{ }^{\star} \mathrm{S}^{\wedge} \mathrm{Nc}\right)$ & $\mathrm{Nc}$ \\
\hline M1 & 217.9 & 404.6 & 27.68 & $512.3 \mathrm{e}-06$ & 0.8817 & $344.9 \mathrm{e}-06$ & 0.7617 \\
\hline M2 & 38.8 & 752.9 & 24.75 & $673.2 \mathrm{e}-06$ & 0.8398 & $242.0 \mathrm{e}-06$ & 0.720 \\
\hline M3 & 35.38 & 1671 & 54.74 & $135.6 \mathrm{e}-06$ & 0.6979 & $111.4 \mathrm{e}-06$ & 0.6663 \\
\hline M4 & 74.71 & 839.5 & 54.89 & $2.43 \mathrm{e}-03$ & 0.6229 & $562.9 \mathrm{e}-06$ & 0.6525 \\
\hline
\end{tabular}

Fuente: autores.

\section{REFERENCIAS}

[1] G. Bejarano, M. Gómez, J. Osorio , J. Caicedo and W. Aperador, "Tribological properties and corrosion resistance enhancement of $\mathrm{H} 13$ hot work steel by means of CrN/TiN multilayers," Journal of Materials Science and Engineering, vol. 4, no. 6, pp. 51-59, 2010.

[2] M. T. Cortés y P. Ortiz, “Corrosión," Apuntes Científicos Uniandinos, no. 4, Dic. 2004.

[3] P.P. Darja Kek, M. Cekada and M. Macek, "The corrosión behavior of $\mathrm{Cr}-(\mathrm{C}, \mathrm{N}) \mathrm{PVD}$ hard coatings deposited on various substrates," Electrochimica Acta, vol. 49, pp. 1527 - 1533, 2004.

[4] D. Marulanda and J. J. Olaya, "Unbalanced magnetron sputtering system for producing corrosion resistance multilayer coatings," Dyna, vol. 79, no. 171, pp. 74 79, 2012.

[5] P. H. Mayrhofer, H. Willimann, C. Mitter, "Oxidation kinetics of sputtered $\mathrm{Cr}-\mathrm{N}$ hard coatings," Surface and Coatings Technology, no. 146 - 147, pp. 222 - 228, 2001.

[6] A. Matthews and A. Leyland, "Developments in PVD tribological coatings", HEAT TREAT, vol. 28, no.3, pp. 63-70, 2001

[7] X. Nie, A. Leyland, A. Matthews, "Low temperature deposition of $\mathrm{Cr}(\mathrm{N}) / \mathrm{TiO} 2$ coatings using a duplex process of unbalanced magnetron sputtering and micro-arc oxidation," SURF COAT, no.133, pp. 331-337, 2000.

[8] K.S. Fancey, A. Leyland, F.M. Badow, A. Matthews, "The influence of neon in the deposition of titanium nitride by plasma-assisted physical vapour deposition," MAT
SCI E A, vol. 262, no.1-2, pp. 227-231, 1999.

[9] A. Leyland, M. Binsudin, A.S. James, M.R. Kalantary, P.B. Wells, A. Matthews, J. Housden, B. Garside, "TiN and CrN PVD Coatings on electroless nickel-coated steel substrates," Surface \& Coatings Technology, vol. 60, no.1-3, pp. 474-479, 1993.

[10] D. García, U. Piratoba, “(Ti, Al) N coatings on AISI 4140 by r.f. sputtering," DYNA, no.15, 181-185, 2007.

[11] H.C. Barshilia, K.S. Rajam, "Performance evaluation of reactive direct current unbalanced magnetron sputter deposited nanostructured TiN coated high-speed steel drill bits," Bulletin Materials Science, vol. 30, pp. 607614, 2007.

[12] A. Singh, M. Geetha, N. Kumar, P. Kuppusami, R. Thirumurugesan, E. Mohandas, V. Kamaraj, "A comparative wear study of sputtered ZrN coatings on Si substrate," Transactions of the Indian Institute of Metals, vol. 64, no. 1-2, pp. 37 - 40, 2011.

[13] J. Meghani, K. B. Pai, M. K. Totlani, N. Jalgoankar, "Corrosion and wear behavior of $\mathrm{ZrN}$ thin films," Proceedings of the world congress on engineering, vol. 3, 2010.

[14] O.M. Quintero, A.C Chaparro, L. Ipaz, J.E Sánchez Barco, F. Espinoza Beltrán, G. Zambrano, "Influence of the microstructure on the electrochemical properties of AlCr-N coatings deposited by co-sputtering method from a Cr-Al binary target," Materials Research, São Carlos, vol. 16, falta número, pp, faltan páginas, 2013.

[15] W. Aperador, A. Delgado, J. Duque, "Corrosion resistanne of the [TiN/CrN]n coatings deposited on steel AISI 4140," International Journal of Electrochemical Science, vol. 8, pp. 10711 - 10719, 2013. 
[16] J.J. Zhang, M.X. Wang, J. Yang, Q.X. Liu, D.J. Li, “Enhancing mechanical and tribological performance of multilayered CrN/ZrN coatings," Surface and coatings technology, no. 201, pp. 5186-5189, 2007.

[17] Z.J. Liu, P.W. Shum, Y.G. Shen, "Hardening mechanisms of nanocristalline Ti-Al-N solid solution films," Thin Solid Films, no. 468, pp. 161-166, 2004.

[18] H. Cifuentes and J.J. Olaya, "Microestructure and corrosión resistance of chromium nitrides obtained by vacuum gas nitriding of electrolytic chromium deposite don AISI H13 steel," Revista de Ingeniería Unviersidad de Medellín, vol. 12, no. 22, pp 157 - 167, 2013.

[19] D.D. Macdonald, "Electrochimical," Acta, no.51, pp. 1376-1388, 2006.

[20] D. A. Jones, "Electrochemical Kinetics of corrosion", in Principles and prevention of corrosion. 2nd Ed. Upper Saddle River NJ. Prentice hall, pp. 94, 1996.
[21] A.S. Bondarenko, "Analytica Chimica," Acta, no. 743, pp. 41- 50, 2012.

[22] D.D. Macdonald, "Some advantages and pitfalls of electrochemical impedance spectroscopy," Corrosion Science, vol. 46, no. 3, pp 229-240, 1990.

[23] V.A. Alves, A.M Chiorcea Paquim, A. Cavaleiro, C.M.A Brett, "The nanostructure and microstructure of steels: Electrochemical Tafel behaviour and atomic force microscopy," Corrosion Science, no. 47, pp. 28712882, 2005.

[24] H.J. Flitt, D.P. Schweinsberg / Corrosion Science 47 (2005) 3034-3052

[25] D. F. Rojas, "Síntesis y caracterización de películas delgadas por sputtering de un blanco de cromo a vacíos medios," Tesis de grado, Esc. Física, UPTC, Tunja, Colombia. 2008. 